\title{
Study on Control Strategy of High Power Multiple Connection Rectifier
}

\author{
Jiao Wen-liang ${ }^{\mathrm{a}, \mathrm{b}}$, Wang Xu-dong ${ }^{\mathrm{a} *}$, SUI Xin ${ }^{\mathrm{a}}$ \\ ${ }^{\text {a }}$ Harbin University of Science and Technology, Harbin No. 52 Xuefu Road, Nangang District, Harbin 150027, China \\ ${ }^{\mathrm{b}}$ Heilongjiang University of Science and Technology, Harbin No. 2468 Puyuan Road, Songbei District, Harbin 150027, \\ China
}

*Corresponding Author: wxd6158@163.com

\begin{abstract}
High power DC speed control power supply which can improve the power factor is a kind of multiple connection rectifier using sequential control, but it will bring the grid large harmonic which cannot be ignored in shallow control. To solve this problem, wave and theory of the input side current, harmonic, total factor of harmonic distortion, fundamental wave factor and power factor are contrastively analyzed in this paper. Moreover, the best control strategy of reducing harmonic is also proposed through the calculating formulas. For the control of high power multiple connection rectifier, the best control strategy is to use sequential control in depth control and synchronous control (parallel control) in shallow control which was proved by experiment. In this method, harmonic is greatly reduced but the power factor is the same.
\end{abstract}

Keywords: power factor, multiple connection, sequential control, harmonic.

\section{Introduction}

In the field of high power DC speed control power supply, in order to improve the grid power factor and reduce the motor current ripple, multiple connection rectifiers are always used to supply electricity for DC motor. Such as 12-phase, 18-phase and 24-phase power rectifier ${ }^{[1-}$ 5]. Many other literatures searched varietals topology structure for improving the power factor. But those methods just increased the pulse number of rectifier, never discussed or analyzed the control method ${ }^{[6-8]}$. They just mentioned sequential control and synchronous control (parallel control), neither did they show quantitative analysis or measurement of harmonic. They also didn't discuss the control method of rectifier ${ }^{[9]}$. They could not give the best control strategy because there was no precise quantitative analysis for the current harmonic in the rectifier side which used multiple connection and sequential control method. The theoretical analysis and practical tests show that the power factor can be improved when we adopt depth control for multiple connection sequential control rectifier, but large harmonic will harm the grid in shallow control. This paper will introduce the best control strategy by quantitively analyzed the power factor and harmonic in different control level of multiple connection rectifier. At last, the research will be proved by experiment.

\section{Harmonic current quantities analysis of multiple connection rectifier in different control methods}

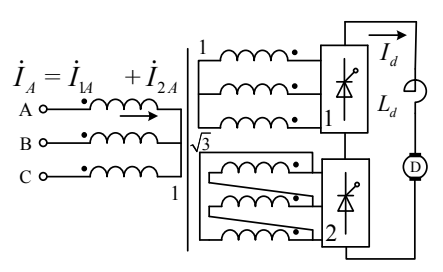

Fig. 1. Inverter main circuit

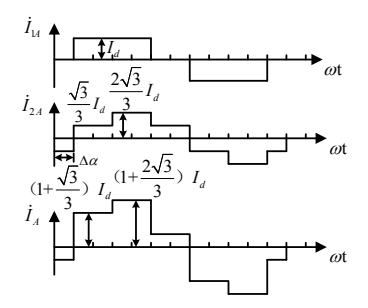

Fig. 2. $\alpha_{1}=0^{\circ}, \alpha_{2}=30^{\circ}$ input side current waveform 
Generally, to obtain a 12-pulse rectifier output voltage, we often use two three-phase full-controlled rectifier circuits in series circuit, also called reconnection rectifier, whose main circuit wiring is shown in Figure.1. The transformer ratio of rectifier transformer is $1: 1: \sqrt{3}$, the line current of input side is $\dot{I}_{A}$, and its values is equal to the sum of $\dot{I}_{I A}$ and $\dot{I}_{2 A}$, which is winding of output side converted to the input side .In order to facilitate the analysis, it is assumed that the rectifier transformer leakage reactance is 0 , inductance of flat wave reactor is infinity. Assuming 1bridge firing angle is $\alpha_{1}, 2$ bridge firing angle is $\alpha_{2}$, $\Delta \alpha=\alpha_{2}-\alpha_{1}$. The common control method is : $\alpha_{1}$ fixed , $\alpha_{2}$ continuously changing or fixed. Corresponding to the input side of the current is shown in Figure 2. Then:

$$
\begin{aligned}
\dot{I}_{1 A}= & \frac{2 \sqrt{3}}{\pi} I_{d}\left(\sin \omega t-\frac{1}{5} \sin 5 \omega t-\frac{1}{7} \sin 7 \omega t+\frac{1}{11} \sin 11 \omega t+\right. \\
& \left.\frac{1}{13} \sin 13 \omega t-\frac{1}{17} \sin 17 \omega t-\frac{1}{19} \sin 19 \omega t+\cdots\right) \\
\dot{I}_{2 A}= & \frac{2 \sqrt{3}}{\pi} I_{d}\left[\sin (\omega t-\Delta \alpha)+\frac{1}{5} \sin 5(\omega t-\Delta \alpha)+\frac{1}{7} \sin 7(\omega t-\Delta \alpha)\right. \\
+ & \frac{1}{11} \sin 11(\omega t-\Delta \alpha)+\frac{1}{13} \sin 13(\omega t-\Delta \alpha)+\frac{1}{17} \sin 17(\omega t-\Delta \alpha) \\
+ & \left.\frac{1}{19} \sin 19(\omega t-\Delta \alpha)+\cdots \cdot \cdot\right]
\end{aligned}
$$

In the formulas: $I_{d}$ is rectifier output DC current, $\omega$ is the power Angular frequency here $100 \pi$.

Applying the principle of superposition to get the total current of network side:

$$
\begin{aligned}
\dot{I}_{A}= & \dot{I}_{I A}+\dot{I}_{2 A}=\frac{4 \sqrt{3}}{\pi} I_{d}\left[\cos \frac{\Delta \alpha}{2} \sin \left(\omega t-\frac{\Delta \alpha}{2}\right)-\frac{1}{5} \sin \frac{5 \Delta \alpha}{2}\right. \\
& \cos \left(5 \omega t-\frac{5 \Delta \alpha}{2}\right)-\frac{1}{7} \sin \frac{7 \Delta \alpha}{2} \cos \left(7 \omega t-\frac{7 \Delta \alpha}{2}\right)+\frac{1}{11} \cos \frac{11 \Delta \alpha}{2} \\
& \sin \left(11 \omega t-\frac{11 \Delta \alpha}{2}\right)+\frac{1}{13} \cos \frac{13 \Delta \alpha}{2} \sin \left(13 \omega t-\frac{13 \Delta \alpha}{2}\right)-\frac{1}{17} \sin \\
& \frac{17 \Delta \alpha}{2} \cos \left(17 \omega t-\frac{17 \Delta \alpha}{2}\right)-\frac{1}{19} \sin \frac{19 \Delta \alpha}{2} \cos \left(19 \omega t-\frac{19 \Delta \alpha}{2}\right) \\
& +\cdots \cdots
\end{aligned}
$$

Formula (2) shows the input side current of the rectifier transformer by sequential control, the 5, 7, 17, $19 \ldots$ harmonics still exist, the amplitude of all harmonics are related to $\Delta \alpha$.

Harmonic current content is the key to harmonic analysis.

The harmonic content of the current $I_{H}$ is:

$$
I_{H}=\sqrt{I_{A}^{2}-I_{1}^{2}}
$$

Valid values of fundamental current:

$$
I_{1}=\frac{2 \sqrt{6}}{\pi} \cos \frac{\Delta \alpha}{2}
$$

Then we can obtain $I_{H}$ by calculating the valid values of the input side line current $I_{A}$.
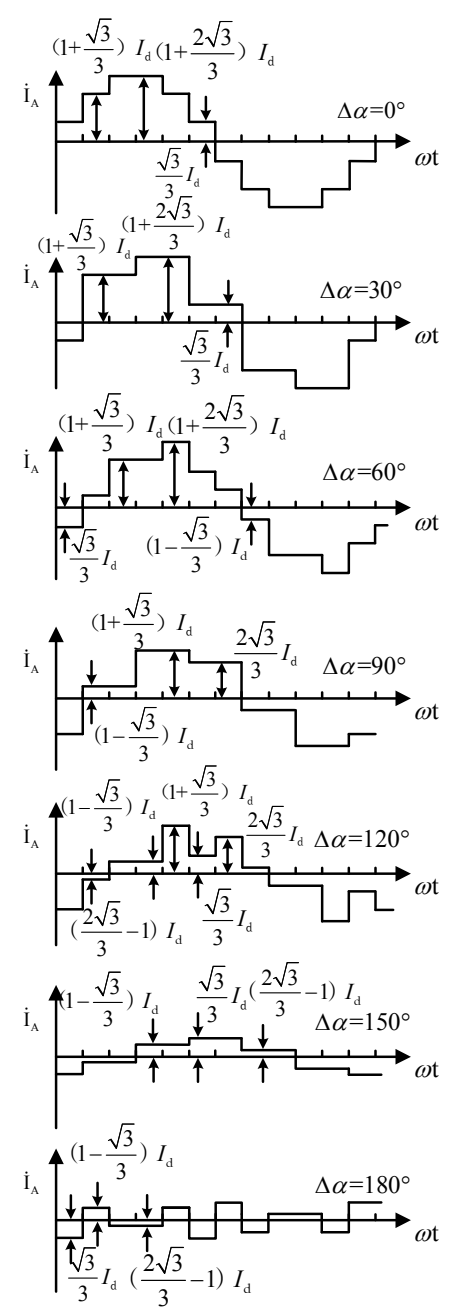

Fig.3. Different $\Delta \alpha$ the net side current waveform

$I_{A}$ is the sum of two winding current in output side of the rectifier transformer, since $\alpha_{1}$ is fixed in the control process, while $\alpha_{2}$ adjusted continuously, the current waveform of $I_{A}$ also changes the same time. The waveforms of $I_{A}$ as $\Delta \alpha$ take different angles current are shown in Figure. 3. The waveforms in Figure 3 shows that, although shapes of $I_{A}$ are different when $\Delta \alpha$ range from 0 to $30^{\circ}$, but each current has the same effect time within a half cycle time.

When $\Delta \alpha=0 \circ,(\sqrt{3} / 3) I_{d}$ and $(1+\sqrt{3} / 3) I_{d}$ appear twice each, continue $60^{\circ},(1+2 \sqrt{3} / 3) I_{d}$ appear once, continue $60^{\circ}$.

When $\Delta \alpha=30^{\circ},(\sqrt{3} / 3) I_{d} 、(1+\sqrt{3} / 3) I_{d} 、(1+2 \sqrt{3} / 3) I_{d}$ appears once each, continue $60^{\circ}$, it is obvious:

When $\Delta \alpha$ ranges from 0 to $30^{\circ}$, valid values of $I_{A}$ is constant.

When $0^{\circ} \leq \Delta \alpha \leq 30^{\circ}$, 


$$
\begin{aligned}
I_{A} & =\sqrt{\frac{1}{\pi} \int_{0}^{\pi} i_{A}^{2} d(\omega t)} \\
& =\sqrt{\frac{1}{\pi}\left[\left(\frac{\sqrt{3}}{3} I_{d}\right)^{2} \times \frac{\pi}{3}+\left(1+\frac{\sqrt{3}}{3}\right)^{2} I_{d}^{2} \times \frac{\pi}{3}+\left(1+\frac{2 \sqrt{3}}{3}\right)^{2} I_{d}^{2} \times \frac{\pi}{3}\right.} \\
& =\sqrt{\frac{4+2 \sqrt{3}}{3}} I_{d} \approx 1.577 I_{d}
\end{aligned}
$$

When $\Delta \alpha$ ranges from $150^{\circ}$ to $180^{\circ}$, the variation regularity of $\dot{I}_{A}$ in a half cycle is the same to when $\Delta \alpha$ range from 0 to $30^{\circ}$.

When $150^{\circ} \leq \Delta \alpha \leq 180$,

$I_{A}=\sqrt{\frac{1}{\pi} \int_{0}^{\pi} i_{A}^{2} d(\omega t)}=\sqrt{\frac{4-2 \sqrt{3}}{3}} I_{d} \approx 0.423 I_{d}$

Through above analysis, we can obtain that the valid values of input side is a constant current when $\Delta \alpha$ in interregional of $0^{\circ} \sim 30^{\circ}$ and $150^{\circ} \sim 180^{\circ}$.

When $30^{\circ}<\Delta \alpha<90$,

$I_{A}=\sqrt{\frac{1}{\pi} \int_{0}^{\pi} i_{A}^{2} d(\omega t)}=\sqrt{\frac{4}{3}-2 \sqrt{3}\left(\frac{\Delta \alpha}{\pi}-\frac{1}{2}\right)} I_{d}$

When $90^{\circ}<\Delta \alpha<150^{\circ}$,

$I_{A}=\sqrt{\frac{1}{\pi} \int_{0}^{\pi} i_{A}^{2} d(\omega t)}=\sqrt{\frac{4}{3}-2 \sqrt{3}\left(\frac{\Delta \alpha}{\pi}-\frac{1}{2}\right)} I_{d}$

By the above analysis, we can know the valid values of current are equal within two intervals,

$I_{A}=\sqrt{\frac{4}{3}-2 \sqrt{3}\left(\frac{\Delta \alpha}{\pi}-\frac{1}{2}\right)} I_{d}$

Put the formula (4), (7) into (3), it an be drawn that when different $\Delta \alpha$,

$I_{\mathrm{H}}=\sqrt{\frac{4+2 \sqrt{3}}{3}-\frac{24}{\pi^{2}} \cos ^{2} \frac{\Delta \alpha}{2} I_{d}}$

Depicts $I_{H}=\mathrm{f}(\Delta \alpha)$ curve as Figure 4 according to (8). As we can see in Figure 4, the content of harmonic current maximum for $0.468 I d$ when $\Delta \alpha=30^{\circ}$, the smallest in $\Delta \alpha=$ $150^{\circ}$. Its value is $0.125 \mathrm{Id}$. $\Delta \alpha$ ranges from $30^{\circ} \sim 150^{\circ}$ wave trough and peaks are at $65.08^{\circ}$ and $114.92^{\circ}$, The corresponding unit values of the harmonic content are 0.291 and 0.388 .

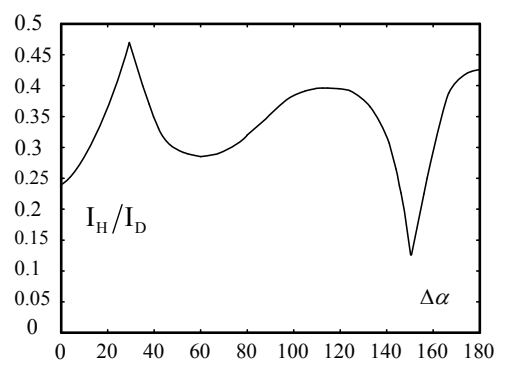

Fig. 4. $I_{H}=\mathrm{f}(\Delta \alpha)$ curve

\section{Power factor analysis of multiple connection sequential control in different control methods}

The power factor of dual connection sequence control rectifier: $\lambda=\xi \cos \varphi, \xi$ is fundamental wave factor, $\xi=I_{I} / I_{A}$.

According to (4), (5), (6), (7), the value of $\xi$ can be determined corresponding to different $\Delta \alpha$, fundamental wave factor $\xi$ change in the range of $0 \sim 0.989$., the $\varphi$ in $\cos \varphi$ can be represented by following formula:

$\varphi=\frac{\alpha_{1}+\alpha_{2}}{2}$

In general, the firing angle of one bridge is fixed, then, variation of the other in the sequence control, assuming that $\alpha_{1}$ fixed, $\alpha_{2}$ changes, then,

$\varphi=\alpha_{1}+\frac{\Delta \alpha}{2}$

The power factor is:

$\lambda=\frac{I_{1}}{I_{A}} \cos \left(\alpha_{1}+\frac{\Delta \alpha}{2}\right)$

The power factor of each segment can be obtained as follow,

When $0^{\circ} \leq \Delta \alpha \leq 30$,

$\lambda=\frac{6}{\pi} \times \frac{\cos \frac{\Delta \alpha}{2} \cos \left(\alpha_{1}+\frac{\Delta \alpha}{2}\right)}{\sqrt{2+\sqrt{3}}}$

When $30^{\circ}<\Delta \alpha<150^{\circ}$,

$\lambda=\frac{6}{\pi} \times \frac{\cos \frac{\Delta \alpha}{2} \cos \left(\alpha_{1}+\frac{\Delta \alpha}{2}\right)}{\sqrt{2-3 \sqrt{3}\left(\frac{\Delta \alpha}{\pi}-\frac{1}{2}\right)}}$

When $150^{\circ} \leq \Delta \alpha \leq 180^{\circ}$,

$\lambda=\frac{6}{\pi} \times \frac{\cos \frac{\Delta \alpha}{2} \cos \left(\alpha_{1}+\frac{\Delta \alpha}{2}\right)}{\sqrt{2-\sqrt{3}}}$

Make two control modes rectified output the same voltage. Compare synchronous and sequential control of the dual connection rectifier power factor.

Make $U_{d T}, U_{d S}$ respectively output voltage of

Synchronous control and sequence control, then,

$U_{d T}=U_{d o} \cos \alpha$.

In the above formula, $\alpha$ is the firing angle of synchronous control,

Let $U_{d T}=U_{d S}$, then,

$\cos \alpha=\frac{\cos \alpha_{1}+\cos \alpha_{2}}{2}=\frac{\cos \alpha_{1}+\left(\cos \alpha_{1}+\Delta \alpha\right)}{2}$ 
Thus we can obtain the power factor of synchronous control, which is equivalent to the sequential control:

$\lambda_{T}=\frac{3}{\pi} \times \frac{\cos \alpha_{1}+\cos \left(\alpha_{1}+\Delta \alpha\right)}{\sqrt{2+\sqrt{3}}}$

According to (12), (13), (14) and (15), we can draw curves of the two power factor control mode as $\alpha_{1}=0^{\circ}$ and $\alpha_{1}=30^{\circ}$ which is shown in Figure. 5 .

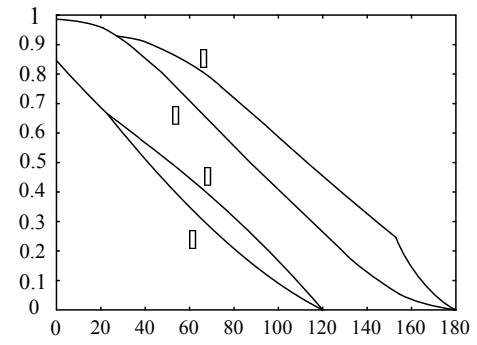

Fig. 5. The power factor of two kinds of control mode curve

(1) sequence control $\alpha_{1}=0^{\circ}$

(2) sequence control $\alpha_{1}=30^{\circ}$

(3) synchronization control $\alpha_{1}=0^{\circ}$

(4) synchronization control $\alpha_{1}=30^{\circ}$

Through the curve in Figure.5., it can be obtained that, there is a large different between two power factors as $\alpha_{1}=0^{\circ}$ and $\Delta \alpha>30^{\circ}$, the maximum difference appears when $\Delta \alpha=150^{\circ}$, its value is 0.18 . While $\alpha_{1}=30^{\circ}$, the maximum difference is 0.072 .

\section{The optimal control strategy}

Generally, if there is requirement for electrical equipment in a reversible operation, we need to set the minimum inverter angle in order to prevent the inverter fails, minimum trigger often take as follow: $\alpha_{\min }=\beta$ min $=30^{\circ}$. $\Delta \alpha$ in this case is often about $30^{\circ}$, it can be seen from the preceding analysis of the power factor and harmonic content that the harmonic of the sequence control is maximum in this case and the value of it is 0.468 , assuredly, the harm to grid is large in this situation . Meanwhile, if sequence control becomes synchronization control, that is to say $\Delta \alpha=0^{\circ}$, then the harmonic content is 0.24 , harmonic content is reduced by $97 \%$. while the power of sequence control and synchronization control is the same when $\Delta \alpha \leq$ $30^{\circ}$, there is no doubt that synchronous control is the best control strategy at this time. $\Delta \alpha$ can take $150^{\circ}$ when motor is running at low speed, then the harmonic content is only 0.125 .

\section{Simulation and experiment}

In order to verify the theoretical analysis, using matlab software do the dual connection circuit simulation analysis. The simulation circuit is shown in Figure 6. System parameters are as follows: supply voltage $380 \mathrm{~V}$, load $10 \Omega, 0.1 \mathrm{mH}$. The simulation results shown in Figure 7, is percentage of each small figure at is the grid current waveform, the harmonic content of 1-50 bar graph below is the grid current, the unit content of the fundamental current.

By analyzing simulation results it can be obtained that, grid current harmonic content is the smaller than in other situations, when $\alpha_{1}=\alpha_{2}=30^{\circ}$, while $\alpha_{1}=30^{\circ}, \alpha_{2}=$ $60^{\circ}$, the grid current harmonic content is largest.

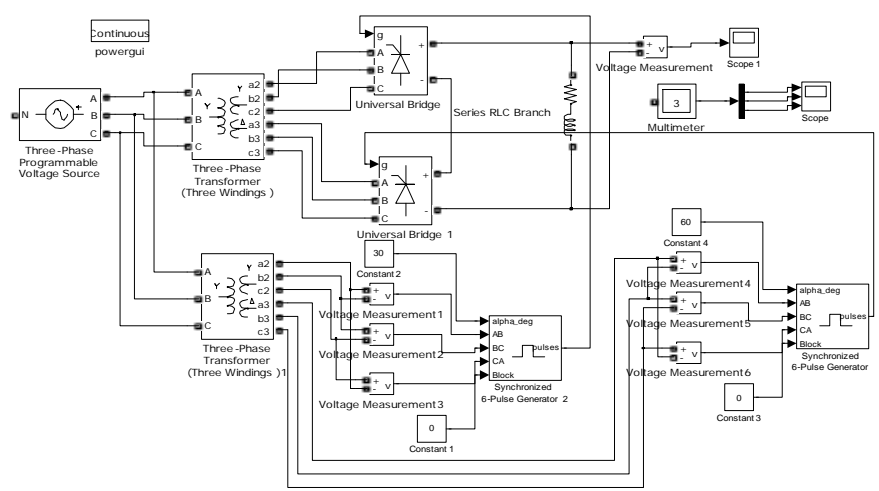

Figure .6. Double connection circuit simulation model
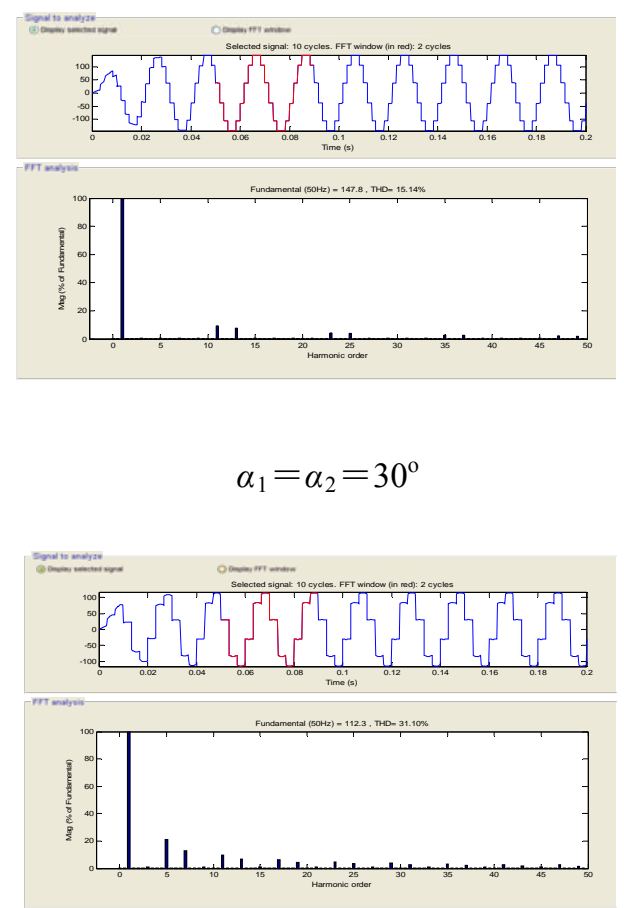

$\alpha_{1}=30^{\circ}, \alpha_{2}=60^{\circ}$ 


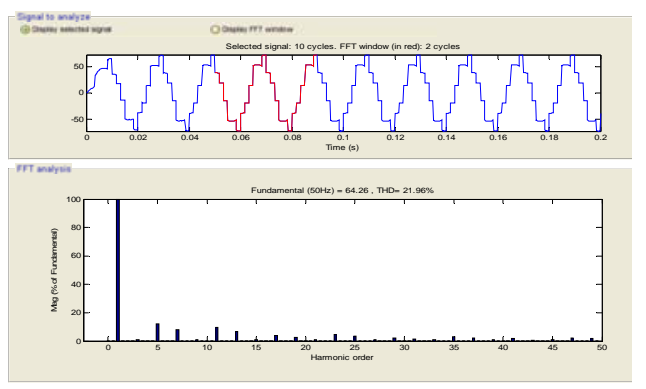

$\alpha_{1}=30^{\circ}, \alpha_{2}=90^{\circ}$

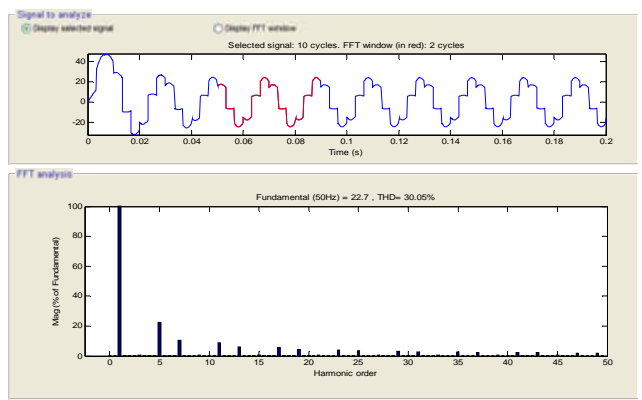

$\alpha_{1}=30^{\circ}, \alpha_{2}=120^{\circ}$

Figure .7. Different $\Delta \alpha$ input current waveform and harmonic content analysis

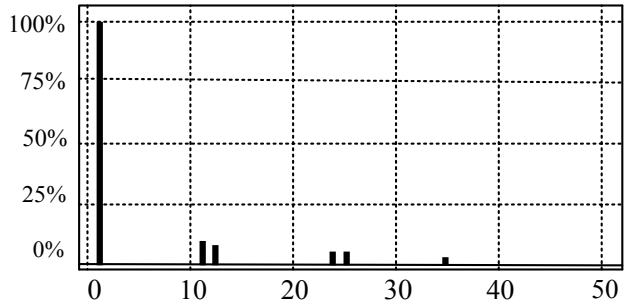

$\Delta \alpha=0^{\circ}$

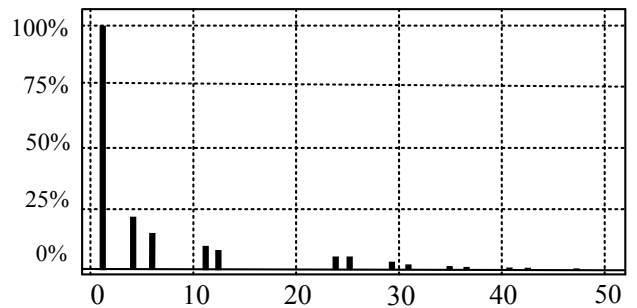

$\Delta \alpha=30^{\circ}$

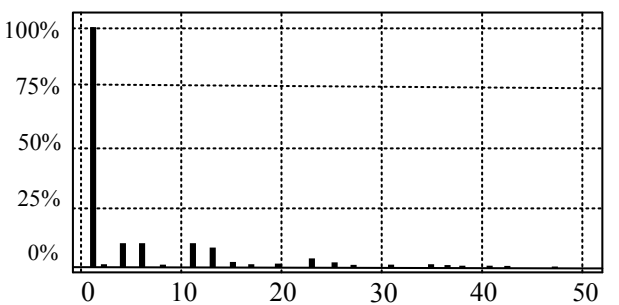

$\Delta \alpha=60^{\circ}$

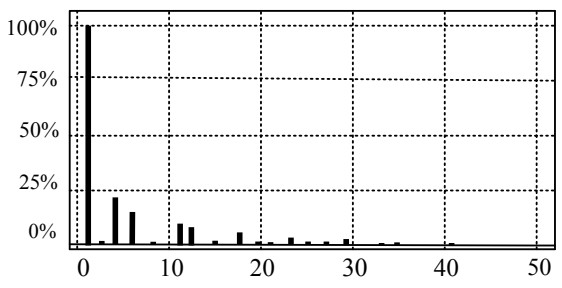

$\Delta \alpha=90^{\circ}$

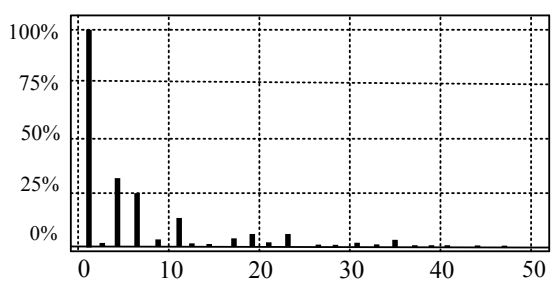

$\Delta \alpha=120^{\circ}$

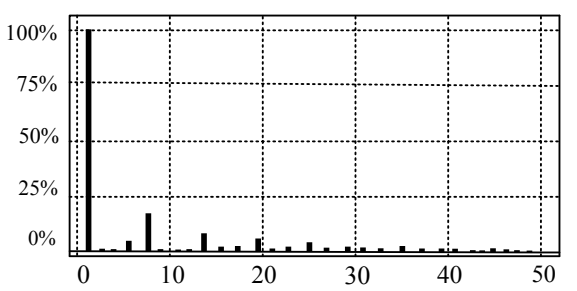

$\Delta \alpha=150^{\circ}$

Figure .8. Measured chart of grid side current harmonics spectrum analysis the of different $\Delta \alpha$

\section{Conclusions}

Through the analysis of connecting multiple sequential control rectifier input harmonic current, power factor and the simulation results we can obtain that, when $\Delta \alpha$ ranges from $0^{\circ}$ to $180^{\circ}$, the harmonic content of the input current is a three continuous function, as $\Delta \alpha=30^{\circ}$, when compared with synchronization control, the harmonic content has 
nearly doubled. When $\Delta \alpha \leq 30^{\circ}$, the power factor of two control modes are equal. Once adopt synchronous control, the power factor basically unchanged, while the harmonic has been greatly reduced, so for the high-power direct current power supply with multiple connection speed circuit, the best control strategy is sequential control when in deep control and synchronous control in shallow control. This control strategy under the premise of not reducing the power factor, greatly reduces the grid current harmonic content, reducing the harmonic pollution to the grid, but also increases the life of the motor, and also improves the speed performance.

\section{References}

(1) Fang Chang-shi, Fang Joshua : "Study on the Double Connection of Modified Bridge Rectifier with Power Factor Improvement", IEEJ Trans, 11, 126, 1479-1485, 2006

(2) Rend Sara D A, von Jouanne A, Enjeti P N, et al. : "Design Considerations for 12-Pulse Diode Rectifier Systems Operating under Voltage Unbalance and Pre-Existing Voltage Di stort ion with Some Corrective Measures", IEEE Trans, 6, 32, 1293-1303, 1996

(3) Farrer W : "Significant Source Harmonic Reduction Achieved Using Direct Parallel Connection of Two 6-pulse Converters", IEE Proc.-Electr. Power Appl., 2, 153, 167-176, 2006

(4) Zargari N R, Xiao Yuan, Wu Bin : "A Multilevel Thyristor Rectifier with Improved Power Factor", IEEE Trans, 5, 33, 1208-1213, 1997

(5) Chivite-Zabalza F J, Forsyth A J, Trainer D R : “A Simple, Passive 24-Pulse AC-DC Converter with Inherent Load Balancing", IEEE Trans. Power Electro., 2, 21, 430-439, 2006

(6) Fang Changshi, Fang Hua: "Research of thyristor with multiphase power factor improving converter", High Power Converter Technology, 6,2009

(7) Hu Wenhua, Song Pinggang : "Features of new dual topology 18-pulse rectifier", High Voltage Engineering, 5,2010

(8) Wang Shitao, lv Zhengyu, Zhang Dehua, Zhang Damin: "A high power factor of multi-pulse thyristor rectifier", Automation of Electric Power Systems,2012.

(9) Bimal K.Bose: "Modern Electronics and AC Drives", Machinery Industry Press, 2013

(10) Rik De Doncker: "Analysis of advanced electric drive, the modeling and control", Machinery Industry
Press, 2013

(11) Yang Yong, Ruan Yi, Ye Binying: "Three-phase grid-connected inverter deadbeat current predictive control method", China Electrical Engineering,33,2009

(12) Wang Shitao, Zhang Damin,Lv Zhengyu: "Thyristor rectifier current controller with fast dynamic response", Journal of Zhejiang University(Engineering Edition),2, 2012

(13) Zhang Yingchao, Zhou Ying: "Windowed Fourier transform in harmonic analysis application", Nanjing University of Information Engineering,6,2003

(14) Seung-Ki Sul: "Motor Drive Control", Machinery Industry Press,2013 